\title{
Seguridad, tecnologías de la información y derechos humanos: impunidad gubernamental e inercia ciudadana*
}

\author{
María Amelia Solórzano Peña** \\ Ramiro Contreras Acevedo***
}

\begin{abstract}
RESUMEN
Este trabajo muestra las contradicciones y fracasos de las politicas públicas de prevención social de la violencia y la delincuencia y del delito. Esto es consecuencia de los excesos e ineficacia en el uso de las tecnologias de la información y comunicación, la impunidad y la inercia del ciudadano que no se percibe parte del fenómeno social de la inseguridad en México. El problema tiene origen en la incorporación acrítica del término seguridad ciudadana a la política pública. El diseño metodológico corresponde al paradigma crítico, mediante una investigación-acción emancipadora-transformadora y a un enfoque metodológico mixto que dará soporte a la argumentación para eliminar los cuestionamientos o atribuciones de subjetividad que se confieren a las investigaciones sociales. La aportación de la investigación al conocimiento existente permite develar la mimetización de lo que hasta ahora se ha entendido como seguridad ciudadana en México y las falsas promesas del trinomio seguridad, tic y derechos humanos.
\end{abstract}

\section{PALABRAS CLAVE}

Violencia, delincuencia, prevención del crimen, tecnologías de la información, violación a los derechos humanos, corrupción, política pública.

\begin{abstract}
This work shows the contradictions and failures of public policies of social prevention of violence and delinquency and crime. This is a consequence of the excesses and inefficiency in the use of Information and Communication Technologies, impunity and the inertia of the citizen that is not perceived as part of the social phenomenon of insecurity in Mexico. The problem originates in the uncritical incorporation of the term Citizen Security into Public Policy. The methodological design corresponds to the critical paradigm, through an emancipatory-transformative research-action and a mixed methodological approach that will support the argumentation to eliminate the questions or attributions of subjectivity that are conferred to social investigations. The contribution of research to existing knowledge allows us to reveal the mimicry of what up to now has been understood as Citizen Security in Mexico and the false promises of the Trinomial Security, ICT and Human Rights
\end{abstract}

\section{KEYWORDS}

Violence, Crime, Crime Prevention, Information Technology, Violation of Human Rights, Corruption, Public Policy

\footnotetext{
*Artículo recibido el 16 de diciembre de 2017 y aceptado para su publicación el 17 de abril de 2018

**Profesora investigadora en la Universidad de Guadalajara, México. (solorzanop@hotmail.com) orcid.org/00000001-6943-5916

***Profesor investigador en la Universidad de Guadalajara, México. (ramirocontreras2000@yahoo.com.mx) orcid. org/0000-0002-7396-8050
} 


\section{SUMARIO}

1. Introducción

2. Los términos seguridad ciudadana y seguridad pública en las políticas públicas

3. El trinomio seguridad -tecnología de la información y la comunicación- derechos humanos

4. Impunidad gubernamental e inercia ciudadana. ¿Fenómenos aislados?

\section{Introducción}

La reflexión que queremos presentar en estas páginas se centra en la fallida vinculación que se pretendió realizar en México del término seguridad ciudadana en las políticas públicas con el uso de las tecnologías de la información y la comunicación (TIC) y los derechos humanos. Este fue el resultado de las reformas estructurales de carácter neoliberal, que son "consecuencia de los pactos con organismos financieros internacionales, acuerdos comerciales o de seguridad con el exterior". ${ }^{1}$ Pese a las evidencias de ausencia del Estado de derecho, del sometimiento a los intereses de otros países o de organismos de financiación internacional, el ciudadano mexicano permanece "inerte, apático e inactivo" y no se percibe como parte del fenómeno de la inseguridad.

Para visibilizar el significado que se le dio a seguridad ciudadana, se decidió partir de algunas opiniones de expertos en el encuadre epistemológico que desde el neoliberalismo se le ha atribuido a la seguridad o inseguridad. Un régimen neoliberal "coloca al mercado, la libertad individual y la desigualdad en el centro de las relaciones sociales, también instaura la idea de escasez, el deseo de competencia, la diferenciación y el miedo al otro en el centro de las subjetividades". ${ }^{2}$ Según estos modos de ver tales fenómenos, se ha de enfatizar la potencialidad del riesgo, la necesidad de implementar medidas preventivas para actuar en defensa de la vida, la libertad y la propiedad. Se alienta la victimización, el estado de alerta permanente, la significación del miedo y la desconfianza en el otro. ${ }^{3}$

Se busca apoyar una combinación de libertad económica con autoritarismo en materia de orden público y seguridad ciudadana, ${ }^{4}$ misma que funciona

\footnotetext{
${ }^{1}$ Cárdenas Gracia, Jaime Fernando, El modelo jurídico del neoliberalismo, México, unam, 2016, p. 111.

2 Garcia, Sergio y Ávila, Débora, "Introducción", en Enclaves de riesgo: Gobierno neoliberal, desigualdad y control social, Madrid, Útiles, 2015, p. 17.

${ }^{3}$ Fernández, Mariana Cecllia, "Foucault y la gubernamentalidad en las sociedades de seguridad", Question, vol. 1, núm. 44, p. 88.

${ }^{4}$ Rodriguez, CÉsar y Uprimny, Rodrigo, "¿Justicia para todos o seguridad para el mercado?", en La falacia neoliberal. Critica y alternativas, Colombia, Universidad Nacional de Colombia, 2003, p. 421
} 
como medio de control social y encuentra su "justificación teórica” en la protección de la sociedad y de los individuos, de aquellos identificados como “delincuentes". ${ }^{5}$ Así pues, la seguridad ciudadana, ${ }^{6}$ restringida a la prevención y control de la delincuencia, ha adquirido una preponderante importancia objetiva y subjetiva sobre otros temas sociales: "la lucha contra la pobreza y sus consecuencias sociales y culturales de marginalidad y exclusión es subordinada al carácter criminógeno de aquella, es decir, la lucha contra la pobreza -la inversión social-, no parecería tener por propósito satisfacer los derechos de los pobres sino prevenir que delincan".7

El significado del concepto seguridad ciudadana propuesto desde el encuadre epistemológico del neoliberalismo, busca hacer exigibles las obligaciones impuestas a los Estados que desean obtener acceso a la denominada "cooperación internacional para el desarrollo" ${ }^{8}$ a través de créditos y financiamiento internacional ${ }^{9}$ y no para defender a los sectores vulnerables o proteger a la sociedad civil de un país. ${ }^{10}$

En este contexto, el sometimiento del Estado mexicano a intereses políticos, financieros y económicos internacionales es una constante a lo largo de los últimos treinta años. ${ }^{11}$ Como consecuencia de la "memoria selectiva y eliminación colectiva del pasado político incómodo", se ha desarrollado un modelo neoliberal sui generis, "a la mexicana", ${ }^{12}$ con un "amplio sentido social"13 para

\footnotetext{
${ }^{5}$ Silva Senaroué, Santos Alfonso, Control social, neoliberalismo y derecho penal, Parte III, Perú, Fondo Editorial de la UNMSM, 2002, p. 289.

${ }^{6}$ La Comisión Interamericana de Derechos Humanos (CIDH) señala que "el concepto de seguridad ciudadana es el más adecuado para el abordaje de los problemas de criminalidad y violencia desde una perspectiva de derechos humanos, en lugar de los conceptos de 'seguridad pública', 'seguridad humana', 'seguridad interior' u 'orden público'". Véase Comisión Interamericana de Derechos Humanos, Informe sobre seguridad ciudadana y derechos humanos, oeA, 31 de diciembre, 2009.

${ }^{7}$ De Rementeria, Ibán, "El estado de la seguridad ciudadana", Polis, núm. 11.

${ }^{8}$ La cooperación internacional al desarrollo es fundamentalmente una forma de ayuda con la cual se busca el incremento de la capacidad productiva y el crecimiento económico. No se presta atención especial a factores como las condiciones de vida de los pueblos (salud, educación, vivienda) o la equidad social. Véase Álvarez Orellana, Scarlet MARINA, "Una introducción a la cooperación internacional al desarrollo", REDUR, núm. 10, p. 290.

${ }^{9}$ Cárdenas Garcia, Jaime Fernando, "Las características juridicas del Neoliberalismo", Cuestiones Constitucionales, vol. 32.

${ }^{10}$ Silva Sernaqué, Santos Alfonso, "El neoliberalismo y derecho penal en las sociedades democráticas", Barco de Papel, vol. 2, núm. 2.

${ }^{11}$ La transformación neoliberal en México fue ortodoxa con los lineamientos del Banco Mundial (BM) y del Fondo Monetario Internacional (FMI). Véase CZARNECKI, LUKASZ, "La concepción de la pobreza en el modelo neoliberal: ¿Cómo entender la lucha contra la pobreza en México?", Frontera norte, vol. 25, núm. 49, p. 180.

${ }^{12}$ Un régimen politico neoliberal, autoritario e ilegítimo, corrupto, privatizador, ajeno a los intereses nacionales y ciego ante las apremiantes necesidades de las grandes mayorias de la población mexicana. Véase Solís González, José Luis, "Neoliberalismo y crimen organizado en México: El surgimiento del Estado narco", Frontera Norte, vol. 25, núm. 50. ${ }_{13}$ Peña Nieto afirmó durante la plenaria de la VII Cumbre de las Américas celebrada en Panamá, los días 10 y 11 de abril de 2015 que "el modelo mexicano es neoliberal pero con amplio sentido social".
} 
justificar la imposición del paquete de reformas estructurales ${ }^{14}$ que supuestamente se realizaron para garantizar el ejercicio de los derechos sociales ${ }^{15} \mathrm{y}$ cerrar las brechas de desigualdad social. ${ }^{16}$

Por lo arriba expuesto, queda evidenciado que, en materia de seguridad, el país se ha subordinado al marco de la cooperación bilateral impuesta por Estados Unidos mediante el Acuerdo de la Iniciativa Mérida. ${ }^{17}$ Este estableció la estrategia de cooperación para el desarrollo (cDCS) de la United States Agency for International Development (USAID) que ha aportado más de 50 millones de dólares al Gobierno de México para "mejorar la seguridad ciudadana mediante el desarrollo de modelos de prevención del delito que fortalezcan la resiliencia de las comunidades y atiendan factores de riesgo asociados al involucramiento de los jóvenes en actividades relacionadas con la violencia”. ${ }^{18}$

Hay que hacer notar que aunque existe la recomendación de la CIDH y la asistencia técnica del USAID al Gobierno mexicano mediante el Programa para la Convivencia Ciudadana (PCC), ${ }^{19}$ ni la Constitución Política de los Estados Unidos Mexicanos, ni el Plan Nacional de Desarrollo 2013-2018, ni el Programa Nacional de Seguridad Pública 2014-2018 ni el Programa Nacional de Derechos Humanos 2014-2018 utilizan el término seguridad ciudadana cuando se refieren a la prevención general y especial de los delitos o a las acciones del Estado para garantizar el goce, ejercicio y respeto de los derechos humanos.

\footnotetext{
${ }^{14}$ La reforma con rasgos claramente neoliberales son consecuencia de los pactos con organismos financieros internacionales, acuerdos comerciales o de seguridad con el exterior. Véase Cárdenas Gracia, Jaime Fernando, El modelo jurídico del neoliberalismo, México, unam, 2016, p. 111.

${ }^{15}$ Las reformas propuestas proponen un modelo de nación diferente al consagrado en los principios sociales de la Constitución de 1917 y sus posteriores reformas. Véase Cárdenas Gracia, JaIme Fernando, El modelo jurídico del neoliberalismo, México, unam, 2016, p. 111.

${ }^{16}$ El neoliberalismo "asistencialista" genera políticas de estabilización y ajuste estructural, para crear los programas que se utilizan para "tranquilizar" a la sociedad y evitar los movimientos sociales. CZARNECKI, LuKAsz, "La concepción de la pobreza en el modelo neoliberal: ¿Cómo entender la lucha contra la pobreza en México?", Frontera norte, vol. 25, núm. 49. Disponible en: http://www.redalyc.org/articulo.oa?id=13625606008 .

${ }^{17}$ Objetivos de la Iniciativa Mérida: Mejorar los programas de las agencias de seguridad de Estados Unidos en la vigilancia de su territorio; equipamiento y activos para apoyar a las agencias de seguridad homólogas; tecnología computarizada para fortalecer la coordinación de las fuerzas de seguridad e información entre México y Estados Unidos; tecnologias para aumentar la capacidad de México para recolectar inteligencia para propósitos de orden público. Véase Arámbula Reyes, Alma, Iniciativa Mérida Compendio, México, Dirección de Servicios de Investigación y Análisis de la Subdirección de Política Exterior de la Cámara de Diputados, 2008, p. 7.

${ }^{18}$ United States Agency for International Development (USAID) Prevención de la violencia y la delincuencia, 2016.

${ }^{19}$ Como resultado de las experiencias del Programa para la Convivencia Ciudadana (PCC) se generó un catálogo de 20 publicaciones con base en seis ejes temáticos: jóvenes en riesgo, salud comunitaria, relación gobierno-sociedad, policia comunitaria, comunicación para la prevención y resiliencia comunitaria. Se promueven conceptos, metodologias, herramientas y las denominadas "buenas prácticas".
} 
Sin embargo, para ajustarse a los términos del acuerdo bilateral de la Iniciativa Mérida y guardar cierta "coherencia conceptual" con el discurso del uSAID, el término seguridad ciudadana quedó reducido solamente a un "enfoque", dentro del marco normativo y la política pública de la prevención social de la violencia y la delincuencia. ${ }^{20}$ De igual forma, se introdujo en el diseño de las estrategias para el logro de un Gobierno cercano y moderno, ${ }^{21}$ como objetivo estratégico en el programa de digitalización nacional ${ }^{22}$ y como tema prioritario de las políticas de ciencia, tecnología e innovación (сті). ${ }^{23}$ No obstante, ninguno de esos instrumentos señala qué debe entenderse por dicho término o si el "enfoque" que se le otorga, desde la prevención social de la violencia y la delincuencia, es también aplicable a estas políticas públicas.

Entonces, el problema que se señala en este escrito y que aquí quiere reflexionarse, consiste en que de acuerdo a las tendencias impuestas por organismos internacionales se incorporó acríticamente el término "seguridad ciudadana" a la política pública nacional, dándole o suponiéndole un significado polisémico. Es decir, el problema se originó por la utilización del término "seguridad ciudadana" sin razonar si es factible su introducción y adecuación a nuestra realidad política, económica, jurídica, social, cultural, etc., con consecuencias y gastos enormes.

La seguridad, las TIC y los derechos humanos se señalan como elementos clave, tanto por la Iniciativa Mérida, como por el PND 2013-2018 para "llevar a México a su máximo potencial”. Por tanto, el objetivo de esta investigación es mostrar las contradicciones de dicho trinomio: entre el discurso, la norma y los programas sectoriales, además del alejamiento del Estado de derecho y la inercia del ciudadano que no se percibe parte del problema de la inseguridad. La fallida vinculación entre el término seguridad ciudadana y el trinomio seguridad - TIC - derechos humanos en las políticas públicas mexicanas se tomó como objeto de estudio. El objetivo es desvincular la lectura de la realidad, de los paradigmas y modelos ineficaces que han sido impuestos en forma acrítica e incidir en el proceso de transformación de dicha realidad.

Para lograr este propósito, si bien el sustento teórico de la prevención social de la violencia y la delincuencia conforme al programa mexicano tiene un sustento teórico de base criminológica, ${ }^{24}$ en el ejercicio de elegir el posicionamiento

\footnotetext{
${ }^{20}$ Véanse el artículo 22, fracción I del Reglamento de la Ley General para la Prevención Social de la Violencia y la Delincuencia, México.

${ }^{21}$ Véase "Programa Gobierno Cercano y Moderno 2013-2018", Diario Oficial de la Federación, 30 de agosto, 2013.

${ }_{22}^{2}$ Véase "Estrategia Digital Nacional", Diario Oficial de la Federación, 1 de noviembre, 2013.

${ }^{23}$ Véase "Programa Especial de Ciencia, Tecnologia e Innovación 2014-2018", Diario Oficial de la Federación, 30 de julio, 2014.

${ }^{24}$ La teoria ecológica del delito, la teoría epidemiológica del delito y la teoría de la eficacia colectiva. Véase "Pro-
} 
epistemológico del investigador se optó por los postulados de los estudios críticos de seguridad ${ }^{25}$ con base en la teoría crítica ${ }^{26}$ desde el enfoque de la escuela de Gales $^{27}$ como teoría sustantiva que favorece el abordaje del fenómeno social. El diseño del marco metodológico corresponde al paradigma crítico ${ }^{28}$ que aunado a una investigación-acción ${ }^{29}$ emancipadora ${ }^{30}$-transformadora y a un enfoque metodológico mixto ${ }^{31}$ que diera soporte a la argumentación, ${ }^{32}$ eliminará los cuestionamientos o atribuciones de subjetividad que, en muchas ocasiones, se confieren a las investigaciones sociales.

Finalmente, la aportación de la investigación al conocimiento existente permite develar la mimetización de lo que hasta ahora se ha entendido como

grama Nacional de Prevención Social de la Violencia y la Delincuencia 2014-2018", Diario Oficial de la Federación, 30 de abril, 2014.

${ }^{25}$ Bajo la denominación de "estudios críticos de seguridad" se agrupan varios enfoques y escuelas que comparten la oposición ontológica y epistemológica a la visión clásica de la seguridad. Véase Pérez de Armiño, KARLos, "¿Más allá de la seguridad humana? Desafíos y aportes de los estudios criticos de seguridad", Cursos de derecho internacional y relaciones internacionales de Vitoria-Gasteiz, núm. 1, 2011, p. 263.

${ }^{26}$ La teoría crítica considera que los problemas de seguridad y la manera en que experimentamos la seguridad/ inseguridad no constituyen una realidad objetiva. De modo que las decisiones en materia de seguridad no son ni naturales, ni irrevocables, ni inmutables. Véase Larenas Álvarez, Angie, "La confluencia entre estudios críticos de seguridad y seguridad humana: las dinámicas de inclusión y superación", Relaciones Internacionales, núm. 23, p. 88.

${ }^{27} \mathrm{Ha}$ contribuido a la ampliación de la agenda de seguridad, al posicionar a la persona como el objeto referente de la seguridad ante una gama de amenazas que va desde la violencia hasta la pobreza y la opresión política. Véase Pérez de Armiño, Karlos, "¿Más allá de la seguridad humana? Desafíos y aportes de los estudios críticos de seguridad", Cursos de derecho internacional y relaciones internacionales de Vitoria-Gasteiz, núm. 1, 2011, p. 271.

${ }^{28}$ La investigación realizada desde el paradigma crítico tiene como objetivo la producción de conocimiento útil para la transformación de la realidad. Véase VARGAS BEAL, XAVER, ¿Cómo hacer investigación cualitativa? Una guía práctica para saber qué es la investigación en general y cómo hacerla, con énfasis en las etapas de la investigación cualitativa, México, Etxeta, 2011, p. 13.

${ }^{29}$ Este método, compartido con el paradigma crítico, "sirve al propósito de la construcción de sentido en los términos que a las distintas ciencias sociales les son útiles", debido a que, el interés epistemológico está ubicado en la construcción de conocimiento teórico. Véase VARGAS BEAL, XAVIER, ¿Cómo hacer investigación cualitativa? Una guía práctica para saber qué es la investigación en general y cómo hacerla, con énfasis en las etapas de la investigación cualitativa, México, Etxeta, 2011, p. 37.

${ }^{30}$ La investigación-acción emancipadora se constituye como una herramienta de transformación social dirigida a promover un auténtico desarrollo humano, para logar un conocimiento humanizador y emancipatorio. Véase ToRREGo-EGIDO, LuIs, "¿Investigación difusa o emancipatoria? Participación e inclusión en investigación educativa", Magis, Revista Internacional de Investigación en Educación, vol. 7, núm. 14, p. 123.

${ }^{31}$ La premisa central es que el uso de enfoques cuantitativos y cualitativos en combinación provee una mejor comprensión de los problemas de investigación que cualquier enfoque utilizado independientemente. Véase Sıvvo Donolo, Danilo, "Triangulación: Procedimiento incorporado a nuevas metodologías de investigación", Revista digital Universitaria unam. $m x$, vol. 10, núm. 8, p. 6 .

${ }^{32}$ Respecto a los cuestionamientos del estatus científico y el rigor de los estudios sociales, el criterio de objetividad para el paradigma crítico es el argumento; el discurso es considerado como teoría en la medida en que explique racionalmente la realidad. Véase Melchor Aguilar, Jaime y Martinez Revilla, Anel, "Los Sistemas de Investigación en México", Cinta de Moebio, núm. 14. 
seguridad ciudadana en México, que en el marco de la Iniciativa Mérida ${ }^{33}$ se traduce como "militarización de la seguridad", según la interpretación y adecuaciones ad hoc que cada administración del gobierno estadounidense ha realizado. Esta sumisión ha pasado factura en México, puesto que en 10 años se ha atravesado una crisis de violencia nunca antes vista con índices similares a los de países como Irak, Siria, Nigeria y Afganistán. La Iniciativa ha sido calificada como "la materialización del neoliberalismo", ${ }^{34}$ ya que jurídicamente se fundamenta en acuerdos que comprometen la soberanía nacional, ${ }^{35} \mathrm{e}$ institucionalmente en una estrategia de política exterior de los Estados Unidos ${ }^{36}$ que promueve mecanismos ad hoc de "orden interno" en la región conforme con sus intereses socioeconómicos y geopolíticos. ${ }^{37}$ La lectura de los resultados del acuerdo de cooperación no es optimista con respecto a la medición de indicadores relativos a gobernabilidad, paz, seguridad, Estado de derecho, entre otros, ya que México se ubica por debajo de la media o con tendencia al descenso; incluso, en algunos indicadores los resultados son tan negativos que no le permiten posicionarse en la clasificación. ${ }^{38}$

La deficiente actuación del Estado mexicano, la sumisión a intereses internacionales por encima del de los mexicanos, los excesos de los servidores públicos, las contradicciones y fracasos de las políticas públicas, la corrupción y la impunidad y la exclusión de la participación ciudadana en la toma democrática de decisiones abren una amplia gama de posibilidades para realizar investigaciones que abonen a la producción de conocimiento útil para la transformación integral del país y de sus habitantes.

\footnotetext{
${ }^{33}$ La importancia de este acuerdo de cooperación bilateral no radica realmente en la recepción de recursos, puesto que representan una fracción respecto al gasto público asignado en materia de seguridad. La importancia está en que, sin tener la calidad de tratado internacional, impulsó las reformas constitucionales y legislativas en materia penal que se realizaron en nuestro pais y que fueron impuestas por los Estados Unidos para proteger su estabilidad y seguridad. Véanse Rangel Cortés, Victor Manuel, "Iniciativa Mérida y la reproducción del derecho penal mexicano", en Reforma Judicial, núm. 25-26.

${ }^{34}$ Rangel Cortés, Victor Manuel, "Iniciativa Mérida y la reproducción del derecho penal mexicano", Reforma Judicial, núm. 25-26, p. 85.

${ }^{35}$ Cárdenas Garcia, Jaime Fernando, "Las caracteristicas juridicas del Neoliberalismo", Cuestiones Constitucionales, vol. 32, p. 42.

${ }^{36} \mathrm{Al}$ amparo este acuerdo, los Estados Unidos toman posesión militar del territorio, de la infraestructura y los recursos estratégicos, así como de la conciencia de los mexicanos. Véase Sánchez Sandoval, Augusto, Seguridad nacional y derechos humanos, México, unam, 2013, p. 137.

${ }_{37}$ Delgado-Ramos, Gian Carlo y Romano, Silvina Maria, "Plan Colombia e Iniciativa Mérida: negocio y seguridad interna", El Cotidiano, núm. 170, p. 89.

${ }^{38}$ Las calificaciones de la variable de Estado de derecho del Índice de Gobernabilidad del Banco Mundial siempre han sido negativas, es decir, por debajo de cero, ubicadas en la parte izquierda de la distribución. Véase Rios, VIRIDIANA, Enriouez, Alejandra, Espejel, Omar y Galindo, Mariana, "Estado de Derecho", México ¿cómo vamos?, vol. 1, p. 7.
} 


\section{Los términos seguridad ciudadana y seguridad pública en las políticas públicas}

El Programa Nacional para la Prevención Social de la Violencia y la Delincuencia 2014-2018 ha sido denominado, equivocadamente, "Programa Nacional de Prevención del Delito" (Pronapred). Es una equivocación porque, en primer lugar, constitucionalmente la prevención de los delitos es privativa de la seguridad pública; en segundo, porque ni su fundamento, ni sus objetivos se orientan al delito, a su prevención o la reducción de la incidencia delictiva.

Efectivamente, los significados que el marco jurídico mexicano atribuye a los términos seguridad ciudadana ${ }^{39}$ y seguridad pública ${ }^{40}$ establecen claramente que la diferencia radica en los objetos de estudio que les son atribuidos. La seguridad pública se centra en el delito, mientras que la seguridad ciudadana en las causas que generan violencia y delincuencia, tal y como se desprende del siguiente cuadro comparativo:

Gráfico 1. Cuadro comparativo de los conceptos de prevención del delito y prevención social de la violencia y la delincuencia ${ }^{41}$

\begin{tabular}{|l|l|}
\hline Prevención del delito & $\begin{array}{l}\text { Prevención social de la violencia } \\
\text { y la delincuencia }\end{array}$ \\
\hline $\begin{array}{l}\text { Estrategias y medidas que buscan la } \\
\text { reducción del riesgo de que ocurran delitos, } \\
\text { así como sus efectos dañinos potenciales } \\
\text { en los individuos y la sociedad, incluyendo } \\
\text { el temor al delito, mediante la intervención } \\
\text { para influir en sus múltiples causas. }{ }^{1}\end{array}$ & $\begin{array}{l}\text { Acciones destinadas a atacar las causas } \\
\text { sociales estructurales de la violencia y la } \\
\text { delincuencia, tales como el desempleo, la } \\
\text { desintegración familiar o las carencias } \\
\text { en la educación formal, mediante } \\
\text { programas integrales de desarrollo social, } \\
\text { cultural y económico, incluidos los de salud, } \\
\text { educación, vivienda, empleo y desarrollo } \\
\text { urbano. }{ }^{2}\end{array}$ \\
\hline
\end{tabular}

\footnotetext{
${ }^{39}$ Obligación del Estado de garantizar la seguridad de la persona actuando sobre las causas que originan la violencia, la delincuencia y la inseguridad. Véase: Artículo 22, fracción I del Reglamento de la Ley General para la Prevención Social de la Violencia y la Delincuencia.

${ }^{40}$ Función a cargo de la Federación, las entidades federativas y los municipios que comprende la prevención de los delitos, la investigación y persecución para hacerla efectiva, así como la sanción de las infracciones administrativas, en los términos de la ley, en las respectivas competencias que esta Constitución señala. Véase: Artículo 21 de la Constitución Política de los Estados Unidos Mexicanos, México, 5 de febrero de 1917, Diario Oficial de la Federación: 15 de septiembre de 2017.

${ }^{41}$ Cuadro elaborado con información obtenida del Programa Nacional de Prevención Social de la Violencia y la Delincuencia 2014-2018.
} 
Cuando el operador político no logra establecer con precisión los objetos de estudio de la seguridad pública y la seguridad ciudadana, produce consecuentemente la ambigüedad de los significados de los tipos de prevención y el posterior impacto que tendrían los resultados en la política pública.

En el siguiente gráfico se puede verificar que los objetivos relativos a la prevención social de la violencia y la delincuencia en ningún momento hacen referencia expresa al delito o su prevención, ya que son atribuciones exclusivas en materia de seguridad pública.

Gráfico 2. Cuadro comparativo de los objetivos del Programa Nacional de Seguridad Pública 2014-2018 y el Programa Nacional de Prevención Social de la Violencia y la Delincuencia 2014-2018 ${ }^{42}$

\begin{tabular}{|l|l|}
\hline $\begin{array}{l}\text { Programa Nacional de Seguridad } \\
\text { Pública 2014-2018 }\end{array}$ & $\begin{array}{l}\text { Programa Nacional de Prevención Social de la } \\
\text { Violencia y la Delincuencia 2014-2018 }\end{array}$ \\
\hline $\begin{array}{l}\text { Objetivo 1. Consolidar una } \\
\text { coordinación efectiva para el diseño, } \\
\text { implementación y evaluación de la } \\
\text { política de seguridad } \\
\text { pública. }\end{array}$ & $\begin{array}{l}\text { Objetivo 1. Incrementar la corresponsabilidad de } \\
\text { la ciudadanía y actores sociales en la prevención } \\
\text { social mediante su participación y desarrollo } \\
\text { de competencias. }\end{array}$ \\
\hline $\begin{array}{l}\text { Objetivo 2. Reducir la incidencia de } \\
\text { los delitos con mayor impacto en la } \\
\text { población. }\end{array}$ & $\begin{array}{l}\text { Objetivo 2. Reducir la vulnerabilidad ante la } \\
\text { violencia y la delincuencia de las poblaciones de } \\
\text { atención prioritaria. }\end{array}$ \\
\hline $\begin{array}{l}\text { Objetivo 3. Reducir la incidencia de } \\
\text { los delitos vinculados con la } \\
\text { economía ilegal. }\end{array}$ & $\begin{array}{l}\text { Objetivo 3. Generar entornos que favorezcan la } \\
\text { convivencia y la seguridad ciudadana. }\end{array}$ \\
\hline $\begin{array}{l}\text { Objetivo 4. Desarrollar en las } \\
\text { instituciones de seguridad pública } \\
\text { esquemas de proximidad y cercanía } \\
\text { con la sociedad. }\end{array}$ & $\begin{array}{l}\text { Objetivo 4. Fortalecer las } \\
\text { capacidades institucionales para la seguridad } \\
\text { ciudadana en los gobiernos municipales, } \\
\text { delegacionales, estatales y federal. }\end{array}$ \\
\hline $\begin{array}{l}\text { Objetivo 5. Fortalecer las capacidades } \\
\text { de las instituciones policiales. }\end{array}$ & $\begin{array}{l}\text { Objetivo 5. Asegurar la coordinación entre las } \\
\text { dependencias y entidades de la Administración } \\
\text { Pública Federal para la implementación de } \\
\text { programas de prevención social. }\end{array}$ \\
\hline $\begin{array}{l}\text { Objetivo 6. Fortalecer el sistema } \\
\text { penitenciario nacional y el } \\
\text { especializado en menores de edad que } \\
\text { infringen la ley penal. }\end{array}$ & \multicolumn{2}{|l}{} \\
\hline
\end{tabular}

Ante los nulos resultados del Pronapred, en el 2014, producto de un análisis externo se detectaron errores de diseño en la implementación de estrategias

\footnotetext{
${ }^{42}$ Cuadro elaborado con información obtenida del Programa Nacional de Seguridad Pública 2014-2018 y el Programa Nacional de Prevención Social de la Violencia y la Delincuencia 2014-2018.
} 
y la ausencia de elementos para la evaluación de sus resultados. Además, se acusó la falta de claridad en la metodología de elección de las demarcaciones y en el monto de recursos asignado a cada una. ${ }^{43}$ Posteriormente, se insistió en transparentar los criterios de selección de polígonos, corregir los errores en la metodología y aclarar el destino del gasto, puesto que gran parte del presupuesto se empleó en difusión de contenidos relacionados con el programa: campañas temáticas, entrega de aparatos auditivos, actividades en torno a huertos comunitarios y rehabilitación de espacios públicos como estrategia de prevención situacional del delito. Sin embargo, no se identificó que su implementación fuese producto de diagnósticos que acreditaran la existencia de factores de riesgo y cómo podrían incidir en la prevención del delito o en la disminución en la posibilidad de ser víctima de la violencia y la delincuencia ${ }^{44}$.

Cuando la Auditoría Superior de la Federación (ASF) fiscalizó el desempeño del subsidio Pronapred y de la Comisión Intersecretarial para la Prevención Social de la Violencia y la Delincuencia (CIPSVD), ${ }^{45}$ confirmó que no se identificaron elementos para determinar los efectos de la política pública en la reducción de los factores de riesgo. Además, se comprobó que 57.1\% de los programas (48 de 84), así como 87\% de las líneas de acción (114 de 131), que se financiarían con recursos establecidos en el Anexo 18 del Decreto de Presupuesto de Egresos de la Federación del $2014,{ }^{46}$ no tenían relación con las acciones previstas en el Programa Nacional para la Prevención Social de la Violencia y la Delincuencia 2014-2018.

La inversión de más de 9778 millones asignados durante el periodo 2013 a 2016 no se materializó en una reducción significativa en la incidencia delictiva ${ }^{47}$ o en la percepción de inseguridad de los ciudadanos. ${ }^{48}$ Una vez más, se

\footnotetext{
${ }^{43}$ Chapa Koloffon, Lilan, Fernández Novelo, Leonel y Ley, Sandra, "México Prevención del Delito en México: ¿Dónde Quedó la Evidencia?", México Evalúa, 2014.

${ }^{44}$ Chapa Koloffon, Llian y Ley, Sandra, "Prevención del delito en México: ¿Cuáles son las prioridades?", México Evalúa, 2015.

$290{ }^{45}$ Secretaria de Hacienda y Crédito Públco, "Informe del Resultado de la Fiscalización Superior de la Cuenta Pública 2014 del Dictamen de la Auditoría de Desempeño 14-0-04100-07-0113 'Prevención Social de la Violencia y la Delincuencia'", Secretaría de Gobernación, 23 de octubre, 2015. Disponible en: https://www.asf.gob.mx/Trans/Informes/ IR2014i/Documentos/Auditorias/2014_0113_a.pdf

${ }^{46}$ Presupuesto de Egresos de la Federación para el Ejercicio Fiscal 2014. México, Diario Oficial de la Federación: 3 de diciembre de 2013.

${ }^{47}$ Durante 2016 se generaron 31.1 millones de delitos asociados con 24.2 millones de victimas. Esto representa una tasa de 1.3 delitos por víctima (mismo nivel registrado en 2015). Véase Instituto Nacional de Estadistica y Geografia, Encuesta Nacional de Victimización y Percepción de la Seguridad Pública 2017, Boletín de Prensa núm. 417/17, 26 de septiembre de 2017.

${ }^{48}$ Se estima que $74.3 \%$ de la población de 18 años y más considera que vivir en su entidad federativa es inseguro a consecuencia de la delincuencia. La cifra es estadisticamente superior a las estimadas en ediciones anteriores de
} 
hizo evidente la utilización de términos o modelos impuestos sin realizar los procesos de contextualización, análisis y distinción crítica. La literatura especializada en prevención, ${ }^{49}$ incluso la publicada por el Banco Mundial y el Banco Interamericano de Desarrollo, advierte sobre las características particulares cuando se aplica el modelo de prevención social, ${ }^{50}$ también conocido como "prevención mediante el desarrollo social" que requiere trabajo, compromiso, inversión, supervisión y evaluación continua y cuyos resultados se visualizan a largo plazo. ${ }^{51}$ Sin embargo, aunque la lectura del tipo de prevención hubiera sido la correcta, los resultados obtenidos hasta el momento tampoco han sido representativos, en relación con los factores de riesgo identificados como prioritarios en el Programa Nacional de Prevención Social de la Violencia y la Delincuencia, o sea: los embarazos tempranos, ${ }^{52}$ el consumo y abuso de drogas ilegales,$^{53}$ los ambientes familiares deteriorados o problemáticos, ${ }^{54}$ entre otros. Por ello, paulatinamente, el programa perdió su estatus prioritario. ${ }^{55}$

El montaje respecto al "supuesto cambio de paradigma", adoptado en cada sexenio para reducir el delito, se redujo a la presentación de programas de política pública con nombres distintos, pero siendo los mismos acuerdos de cooperación bilateral, y, naturalmente, los mismos pésimos resultados: ${ }^{56}$ los

\footnotetext{
la encuesta. Véase Instituto Nacional de Estadistica y Geografia, Encuesta Nacional de Victimización y Percepción de la Seguridad Pública 2017, Boletín de Prensa núm. 417/17, 26 de septiembre de 2017.

49 Sagant, Valérie y Shaw, Margaret, International Report 2010 on Crime Prevention and Community Safety: Trends and Perspectives, Canadá, International Centre for the Prevention of Crime, 2010.

${ }^{50}$ La prevención social puede ser un tanto inespecifica en su dimensión de seguridad, debido a que la seguridad sería un efecto a largo plazo del esfuerzo concertado de diversas políticas públicas. Véase Centro de Estudios de Seguridad Ciudadana, "Apoyando la prevención en América Latina", +Comunidad + Prevención, núm. 1, p. 4.

${ }^{51}$ Oficina de las Naciones Unidas contra la Droga y el Delito, Manual sobre la aplicación eficaz de las Directrices para la prevención del delito, Nueva York, Oficina de las Naciones Unidas contra la Droga y el Delito, 2011, p. 15.

${ }^{52}$ En la OCDE, México tiene la más alta tasa de fertilidad en adolescentes, 73.6 bebés vivos por cada mil partos y ésta poco ha cambiado en las últimas décadas. Véase Organización para la Cooperación y el Desarrollo Económicos, "La situación de México", Panorama de la Sociedad 2016. Disponible en: https://www.oecd.org/mexico/sag2016-mexico.pdf ${ }^{53} \mathrm{El}$ consumo de cualquier droga ilegal en personas entre los 12 y 65 años aumentó en los últimos cuatro años. La marihuana y la cocaína son las sustancias que más se consumen en el país. Véase Instituto Nacional de Psiouiatria Ramón de la Fuente Muñiz, Encuesta Nacional de Consumo de Drogas, Alcohol y Tabaco (2016-2017): Reporte drogas, 2017. Disponible en: https://drive.google.com/file/d/1zIPBiYB3625GBGIW5BX0T_YQN73eWhR/view

${ }^{54}$ Más de dos quintas partes de los hombres casados o unidos con mujeres de 15 años o más (10.8 millones) han ejercido algún tipo de violencia contra sus parejas a lo largo de su relación: 40\% de manera emocional, 25.3\% económica, $11.6 \%$ física y 5.3\% sexual. Véase Instituto Nacional de Estadistica y Geografia, "Encuesta Nacional sobre la Dinámica de las Relaciones en los Hogares 2016", Instituto Nacional de Estadística y Geografía. Disponible en: http://www.beta.inegi.org.mx/proyectos/enchogares/regulares/dutih/2016/

${ }^{55}$ En consecuencia, se eliminó la partida correspondiente al Pronapred del anexo 19 del Presupuesto de Egresos de la Federación 2017.

${ }^{56}$ A cinco años de la administración de Peña Nieto, se ha alcanzado 94.31\% de homicidios registrados durante todo el periodo de Calderón.
} 
niveles de violencia son equiparables a países en guerra declarada ${ }^{57}$ y México es considerado entre los diez más violentos del mundo. ${ }^{58}$

Este escenario se convirtió en terreno propicio para que los Estados Unidos aplicaran su política injerencista basada en la lucha contra el narcotráfico, justificada en la cooperación bilateral y bajo el amparo de la Ley de Seguridad Interior que es el equivalente a la Ley de Autorización de Defensa Nacional (HR $1540)^{59}$ norteamericana. Se implementó mediante el modelo de seguridad ciudadana, entendida como la militarización de la seguridad pública ${ }^{60}$ y la implementación del sistema penal acusatorio y oral, que armoniza el modelo penal al de Estados Unidos.

\section{El trinomio seguridad -tecnología de la información y la comunicación- derechos humanos}

El conocimiento es el activo más importante que los bienes de capital y mano de obra existen en una economía del conocimiento. El conocimiento debe ser de gran calidad y sofisticación, de manera que permee en las actividades económicas y sociales. Su razón de ser es la creación, difusión y el uso del conocimiento. Este es el nuevo horizonte rector de las políticas públicas educativas y de producción de las tecnologías de la información y la comunicación. ${ }^{61}$

El modelo de desarrollo económico, en la sociedad del conocimiento, "implica un proceso colaborativo que involucra al gobierno (en su tres niveles), empresas, universidades y sus centros de investigación e instituciones sociales a través de ecosistemas de innovación, por lo que toda esta actividad

\footnotetext{
${ }^{57}$ World Health Organization, World health statistics 2017: monitoring health for the sDgS, Sustainable Development Goals, Geneva, World Health Organization, 2017. Disponible en: http://apps.who.int/iris/bitstream/10665/255336/1 19789241565486-eng.pdf?ua=1

${ }^{58}$ Uppsala Universitet, Uppsala Conflict Data Program, México, Department of Peace and Conflict Research, 2017. Disponible en: http://ucdp.uu.se/\#country/70

${ }^{59}$ The American republic is fractured. The tendency is towards the establishment of a totalitarian State, a military government dressed in civilian clothes. The adoption is tantamount to the militarization of law enforcement, the repeal of the Posse Comitatus Act and the Inauguration in 2012 of Police State USA. Véase ChossudovskY, MicheL, "The Inauguration of Police State USA. Towards a Democratic Dictatorship?", Globalresearch, 1 de julio de 2012. Disponible en: https://www.globalresearch.ca/the-inauguration-of-police-state-usa-2012-obama-signs-the-national-defense-authorization-act/28441

${ }^{60}$ El Estado mexicano, en correspondencia con la Iniciativa Mérida, ha militarizado al pais y acude a la represión, al miedo y al terror como medio de control sistémico. Ceja Martinez, Jorge, "Seguridad ciudadana, militarización y criminalización de las disidencias en México (2006-2012)", Espacio Abierto, vol. 22, núm. 4, p. 681. Disponible en: http://www.redalyc.org/pdf/122/12229041008.pdf

${ }^{61}$ Sánchez, Carlos y Rios, Humberto, "La economía del conocimiento como base del crecimiento económico en México", Enl@ce: Revista Venezolana de Información, vol. 8, núm. 2.
} 
y dinámica denota un creciente interés por vincular conocimiento científico al desarrollo local, regional y nacional, derivando en tendencias que se van consolidando y que suben en el ranking de importancia atribuida a la ciencia y la tecnología". ${ }^{62}$

Esto cobra relevancia debido a la actual vinculación entre seguridad y TIc. Un ejemplo es que el término "seguridad ciudadana" se encuentra presente en las estrategias del Programa para un Gobierno Cercano y Moderno 20132018, posicionado como un objetivo estratégico en la Estrategia Digital Nacional e identificado como tema prioritario en el que las TIC deben funcionar como instrumentos para el logro de los objetivos planteados en esta área por el Programa Especial de Ciencia, Tecnología e Innovación 2014-2018 (Peciti). Sin embargo, ninguno de estos instrumentos señala qué debe entenderse por el mismo o si alguna de las acepciones expuestas en el marco de la prevención social de la violencia y la delincuencia es el aplicable a estas políticas públicas. Inclusive, se infiere que dichos programas sectoriales no están diseñados con la misma visión de lo que debe entenderse por seguridad ciudadana.

Muestra de lo anterior es que, en el Peciti 2014-2018, el tema prioritario, denominado seguridad ciudadana, viene vinculado con los objetivos y la estrategia sectoriales en materia de inteligencia militar, procuración de justicia, sistema penal acusatorio, seguridad integral asociada al turismo y a impulsar las relaciones bilaterales. ${ }^{63}$ Por otra parte, el Programa para un Gobierno Cercano y Moderno 2013-2018, en sus estrategias para la inserción de México en la sociedad de la información y del conocimiento, busca fortalecer la "seguridad ciudadana” utilizando medios digitales. La Estrategia Digital Nacional, en el objetivo seguridad ciudadana, se refiere a la utilización de las Tic para promover la seguridad, y prevenir y mitigar los daños causados por los desastres naturales.

Por otra parte, respecto a la vinculación seguridad - derechos humanos, el Programa de Prevención Social de la Violencia y la Delincuencia califica a la "seguridad como derecho humano". Señala que "la violencia y la delincuencia son situaciones que generan amenazas a los derechos humanos y afectan la construcción de la seguridad ciudadana”. No obstante, el Manual y Protocolo para la Elaboración de Políticas Públicas de Derechos Humanos Conforme a los

\footnotetext{
62 Valenzuela Reynaga, Rodolfo, Moreno Milanes, Maria Dolores y Peimbert Romero, Marlene, "Indicadores de economía basada en el conocimiento en organizaciones de Cd. Obregón, Sonora, México, Revista El Buzón de Pacioli, número especial 74, p. 4.

${ }^{63}$ Cuadro de Alineación de las estrategias de los Programas Sectoriales 2013-2018 con las oportunidades estratégicas del Peciti. Véase "Programa Especial de Ciencia, Tecnología e Innovación 2014-2018", Diario Oficial de la Federación, 30 de julio, 2014.
} 
Nuevos Principios Constitucionales y el Programa Nacional de Derechos Humanos 2014-2018 son omisos en cuanto la utilización del término seguridad.

Los instrumentos de las políticas públicas, los programas y estrategias se pusieron en marcha para atender a problemas nacionales, cuya solución es considerada fundamental para el país. Sin embargo, durante todos estos años no se ha dejado claro qué se entiende por seguridad ciudadana vinculada a la cTr.

Por esta falta de visión y de claridad conceptual, ahora de los términos seguridad ciudadana y economía del conocimiento, sin haber realizado diagnósticos que permitieran dimensionar los retos que implicaba la vinculación de estos programas sectoriales, nuevamente se realizaron falsas promesas y resultados muy poco exitosos. Lo anterior ha propiciado el rezago de México frente a otras naciones en la apropiación y aprovechamiento de las Tic. ${ }^{64} \mathrm{Se}$ mantiene la enorme brecha digital: $42.6 \%$ de la población no es usuaria de internet. ${ }^{65}$ Además, se hizo público el escándalo atribuido al exceso y mal uso de los servicios de inteligencia, por realizar acciones de espionaje a periodistas y activistas a través de las $\operatorname{Tic}^{66}$ y la ineficacia e ineficiencia de la Unidad de Inteligencia Financiera de la Secretaría de Hacienda y Crédito Público (sнсP). Desde su creación en el 2014 a la fecha, los resultados en lo conducente a congelar cuentas e incautar bienes provenientes del narcotráfico, de las redes de corrupción y las bandas criminales, no reflejan ningún impacto en la incidencia delictiva. Esto llevó a la renuncia de su titular, una vez que se publicó el Informe de Evaluación Mutua sobre medidas contra el lavado de dinero y financiamiento al terrorismo en el 2018. Aunado a todo lo anterior, es igualmente innegable la impune violación a los derechos humanos.

Posteriormente, en el 2017 se canceló el apoyo financiero para el Pronapred, se disminuyó significativamente el presupuesto asignado a Plataforma México y al Centro de Investigación y Seguridad Nacional (Cisen), que desarrolla el trabajo de inteligencia civil para el Estado mexicano. De igual forma, los recursos destinados a la promoción de los derechos humanos y combate a la discriminación fueron drásticamente reducidos. ${ }^{67}$ En el 2018, pusieron

\footnotetext{
${ }^{64}$ World Economic Forum, "Networked Readiness Index 2016". World Economic Forum. Disponible en: http://reports. weforum.org/global-information-technology-report-2016/economies/\#economy=MEX

${ }^{65}$ Instituto Nacional de Estadistica y Geografia, "Encuesta Nacional sobre Disponibilidad y Uso de las Tecnologías de la Información en los Hogares 2016", Instituto Nacional de Estadistica y Geografía. Disponible en: http://www.beta. inegi.org.mx/proyectos/enchogares/regulares/dutih/2016/

${ }^{66}$ Ahmed, Azam y Perlroth, Nicole, "'Somos los nuevos enemigos del Estado: el espionaje a activistas y periodistas en México", New York Times, 19 de junio, 2017.

67 "Presupuesto de Egresos de la Federación para el Ejercicio Fiscal 2017", Diario Oficial de la Federación, 30 de noviembre, 2016.
} 
en marcha los lineamientos para el otorgamiento de apoyos a las entidades federativas en el marco del Programa Nacional de Prevención del Delito, ${ }^{68} \mathrm{a}$ fin de reactivar el subsidio a la acciones de prevención social de la violencia y la delincuencia, aunque de forma limitada y condicionada. En cuanto al área de сті, la asignación de recursos en ese ramo se redujo 3.7\% en términos reales respecto del año anterior. ${ }^{69}$

\section{Impunidad gubernamental e inercia ciudadana. ¿Fenómenos aislados?}

El presidente Enrique Peña Nieto afirmó que: "para combatir la corrupción se debe partir de reconocer que es un asunto cultural y se encuentra en el ADN de los mexicanos"70 e invitó a construir una nueva cultura ética en la sociedad mexicana. Si la corrupción es una característica de los mexicanos, la impunidad ya es identificada mundialmente como una particularidad inherente al país. Debido a esta situación, es el cuarto país del mundo y primero de América con mayor índice de impunidad, ${ }^{71}$ que está directamente relacionada con la corrupción y el Estado de derecho.

Pero no sólo están las confusiones conceptuales arriba señaladas; Véase que no hay correlación significativa entre el índice de corrupción y los indicadores de pobreza, producto interno bruto y desigualdad. La impunidad existe independientemente de las condiciones sociales o económicas locales del país. ${ }^{72}$ Por otro lado, respecto a la corrupción, a pesar de la promulgación de la Ley General del Sistema Nacional Anticorrupción ${ }^{73}$ y los avances en la implementación del sistema, descendió 28 posiciones en el Índice de Percepción de la Corrupción en el sector público. Con ello, se ubicó en el último lugar, entre los 35 países que integran la OCDE. Además, se sabe que 51\% de los mexicanos han sobornado a autoridades en el último año para acceder a servicios públicos.

\footnotetext{
68 "Lineamientos para el otorgamiento de apoyos a las entidades federativas en el marco del Programa Nacional de Prevención del Delito", Diario Oficial de la Federación, 24 de enero, 2018.

69 "Presupuesto de Egresos de la Federación para el Ejercicio Fiscal 2018", Diario Oficial de la Federación, 29 de noviembre, 2017.

${ }^{70}$ Discurso presentado en el evento "Los 300 líderes más influyentes de México", el dia 8 de septiembre de 2014.

${ }^{71}$ Le Clerco Ortega, Juan Antonio y Sanchez Lara, Gerardo Rodriguez, Índice Global de Impunidad 2017, México, Universidad de Las Américas de Puebla, 2017.

72 Le Clerco Ortega, Juan Antonio y Sanchez Lara, Gerardo Rodriguez, Índice Global de Impunidad 2017, México, Universidad de Las Américas de Puebla, 2017.

73 "Ley General del Sistema Nacional Anticorrupción", Diario Oficial de la Federación, 18 de julio, 2016.
} 
Respecto a la corrupción, en un estudio realizado en el 2015 por la Universidad Autónoma de México ${ }^{74}$ se identifica la percepción que la población tiene sobre dos fenómenos que le son inherentes, pero a los que no suele asociarse: la corrupción y la impunidad. En lo que respecta a la corrupción, 92\% de los encuestados considera que hay corrupción en el país; 63.6\% asoció la corrupción con las palabras delitos e ilegalidad, 52.6\% con "función de gobierno y malos políticos", 26.2\% con "mentiras, deshonestidad y descontento" y sólo 4.7\% con "personas distintas a servidores públicos". Cuando se les pidió autoevaluarse, en una escala donde 0 es "nada honesto" y 10 es "muy honesto", el promedio de los encuestados se calificó con 7.9. De ellos, 18.3\% aceptó que era común que ellos iniciaran la acción corrupta. En cuanto a la impunidad, los encuestados aceptan haber realizado diversas conductas poco éticas o ilícitas, porque 90\% considera que hay muy pocas posibilidades de ser atrapado.

Los resultados anteriores pueden compararse con otra encuesta sobre corrupción realizada en el $2016 .{ }^{75}$ En ella, 79\% de la población asocia la palabra corrupción con una actividad característica y casi exclusiva de los políticos y los trabajadores del Estado (gobernantes saqueando el erario público, policías pidiendo mordidas para "perdonar" multas y burócratas solicitando "propinas" para facilitar trámites). Así, 96\% considera que participa "poco" o "nada" en actos de corrupción. Los entrevistados se mostraron indignados ante la posibilidad de que sus actos fueran catalogados como "corruptos" (ofrecer una mordida para pasar la verificación, poner un "diablito" para pagar menos luz y contratar a un familiar en el trabajo) y "explicaron" que sus actos son ejemplos de "astucia", "audacia" e "ingenio", una especie de justicia social o redistribución de la riqueza en un contexto de desigualdad, saqueo y abusos históricos.

El panorama no es alentador: a los altos índices de violencia, delincuencia y criminalidad que se registran en nuestro país, se unen los escasos resultados en el cumplimiento de los objetivos de las políticas públicas. A la mala administración de los recursos, se le añade la falta de ética y compromiso de las autoridades a todos los niveles de gobierno amparados en la creencia de que la corrupción y la impunidad son la "regla y no la excepción".

La inercia ciudadana es la reacción lógica en un sistema de inseguridad ciudadana. El riesgo de victimización al que se encuentra expuesto el ciudadano que decide emanciparse de las relaciones de poder de las elites es real. Pero en este caso, el miedo asociado a la percepción de estar en riesgo es al Estado.

\footnotetext{
74 Resultados de la Encuesta de Nacional de Corrupción y Cultura de la Legalidad. Véase "Los mexicanos vistos por sí mismos", Nexos, 1 de noviembre, 2015.

${ }^{75}$ Opciona, Evaluación cuantitativa sobre la corrupción en México, 2016.
} 
Así lo confirman los informes de tortura, las desapariciones forzadas, ejecuciones extrajudiciales y detenciones arbitrarias, la impunidad por violaciones de derechos humanos y crímenes de derecho internacional.

Finalmente, como se ha explicado a lo largo de este documento, esta no es una investigación realizada en los términos de un paradigma positivista de investigación. El propósito no ha sido establecer relaciones de causa y efecto entre variables, ya que no se busca comprobar ninguna hipótesis, ni contribuir a alguna postura teórica o emitir conclusiones con base en resultados empíricos. Por el contrario, lo que se busca es la producción de conocimiento útil para construir soluciones para la transformación de la realidad. Por ese motivo, queda claro que la aportación de esta investigación permite develar la mimetización de lo que hasta ahora se ha entendido como "seguridad ciudadana" en México y las falsas promesas del trinomio "seguridad - TIC - derechos humanos", que se usaron en el discurso, la norma y los programas sectoriales, además del alejamiento del Estado de derecho y la inercia del ciudadano que no se percibe parte del problema de la inseguridad. La exposición de esta "mimetización" permite desvincular la lectura de la realidad, de los paradigmas y modelos ineficaces que han sido impuestos; además, en forma acrítica para poder incidir en el proceso de emancipación de los ciudadanos y en la toma de decisiones para la transformación de su realidad, sin ninguna otra pretensión empírica.

Aunado a lo anterior, el momento histórico no permite todavía cerrar algunas conclusiones. Quizá lo que aquí podría considerarse son reflexiones o referencias a tareas "prometidas": la aprobación express de la Ley de Justicia Interior sin dar voz ni atención a las preguntas y propuestas de organismos internacionales, diversas ong, académicos e investigadores y a los ciudadanos en general, abre una amplia gama de posibilidades para realizar investigación. Las decisiones en materia de seguridad no son ni naturales, ni irrevocables, ni inmutables. ${ }^{76}$ Es posible que, en el corto plazo, sea prioritario realizar investigación respecto a la forma como México deber realizar un cambio radical en la distribución del poder y en la manera en que el poder debe ser concebido y ejercido.

\footnotetext{
${ }^{76}$ Larenas Álvarez, Angie, "La confluencia entre estudios críticos de seguridad y seguridad humana: las dinámicas de inclusión y superación", Relaciones Internacionales, núm. 23. Disponible en: https://revistas.uam.es/rrii/article/ view/5180
} 


\section{Bibliografía}

Ahmed, Azam y Perlroth, Nicole, “'Somos los nuevos enemigos del Estado: el espionaje a activistas y periodistas en México", New York Times, 19 de junio, 2017. Disponible en: https://www.nytimes.com/es/2017/06/19/mexico-pegasus-nso-group-espionaje/?action=clickEtcontentCollection=AmericasCtmodule $=$ TranslationsCtregion=HeaderCtversion=es-LACtref=en-USCtpgtype $=$ article

Amnistía Internacional, "Informe 2016/2017", Amnistía Internacional. Disponible en: https://www.amnesty.org/es/countries/americas/mexico/report-mexico/

“Acuerdo por el que se establecen los Lineamientos para el otorgamiento de apoyos a las entidades federativas en el marco del Programa Nacional de Prevención del Delito”, Diario Oficial de la Federación, 15 de febrero, 2016.

Álvarez Orellana, Scarlett Marina, "Una introducción a la cooperación internacional al desarrollo”, Redur, núm. 10. Disponible en: http://www.unirioja.es/dptos/dd/ redur/numero 10/alvarez.pdf

Arámbula Reyes, Alma, Iniciativa Mérida Compendio, México, Dirección de Servicios de Investigación y Análisis de la Subdirección de Política Exterior de la Cámara de Diputados, 2008. Disponible en: http://www.diputados.gob.mx/sedia/sia/spe/ SPE-CI-A-02-08.pdf

Cárdenas Gracia, Jaime Fernando, El modelo jurídico del neoliberalismo, México, unam, 2016.

Cárdenas García, Jaime Fernando, "Las características jurídicas del Neoliberalismo", Cuestiones Constitucionales, vol. 32. Disponible en: http://www.redalyc.org/ articulo.oa?id=88541038001

Ceja Martínez, Jorge, "Seguridad ciudadana, militarización y criminalización de las disidencias en México (2006-2012)”, Espacio Abierto, vol. 22, núm. 4, p. 681. Disponible en: http://www.redalyc.org/pdf/122/12229041008.pdf

Centro de Estudios de Seguridad Ciudadana, “Apoyando la prevención en América Latina”, + Comunidad + Prevención, núm. 1. Disponible en: http://www.cesc.uchile.cl/ publicaciones/com_y_prev_01.pdf

Centro Internacional para la Prevención de la Criminalidad, "Estrategias y mejores prácticas en prevención del delito con relación a áreas urbanas y juventud en riesgo", Actas del Taller realizado en el $11^{\circ}$ Congreso de las Naciones Unidas sobre Prevención del Delito y Justicia penal, Montreal, 2007.

Chapa Koloffon, Lilian, Fernández Novelo, Leonel y Ley, Sandra, "Prevención del Delito en México: ¿Dónde Quedó la Evidencia?”, México Evalúa, 2014. Disponible en: http://mexicoevalua.org/2014/01/08/prevencion-del-delito-en-mexico-donde-quedo-la-evidencia/

Chapa Koloffon, Lilian y Ley, Sandra, "Prevención del delito en México: ¿Cuáles son las prioridades?", México Evalúa, 2015. Disponible en: http://mexicoevalua. org/2015/06/16/prevencion-del-delito-en-mexico-cuales-son-las-prioridades/

Chossudovsky, Michel, "The Inauguration of Police State USA. Towards a Democratic Dictatorship?”, Globalresearch, 1 de julio, 2012. Disponible en: https://www. 
globalresearch.ca/the-inauguration-of-police-state-usa-2012-obama-signs-the-national-defense-authorization-act/28441

Comisión Interamericana de Derechos Humanos, "Informe sobre seguridad ciudadana y derechos humanos", Comisión Interamericana de Derechos Humanos, 31 diciembre, 2009. Disponible en: http://www.cidh.org/countryrep/seguridad/seguridadindice.sp.htm

CZARnECKI, LuKASz, "La concepción de la pobreza en el modelo neoliberal: ¿Cómo entender la lucha contra la pobreza en México?”, Frontera norte, vol. 25, núm. 49. Disponible en: http://www.redalyc.org/articulo.oa?id=13625606008

De Rementería, IbÁn, "El estado de la seguridad ciudadana”, Revista Polis, núm. 11. Disponible en: http://polis.revues.org/5759

Delgado-Ramos, Gian Carlo y Romano, Silvina María, "Plan Colombia e Iniciativa Mérida: negocio y seguridad interna”, El Cotidiano, núm. 170.

“Estadísticas Oportunas de Finanzas Públicas”, Secretaría de Hacienda y Crédito Público, enero, 2017. Disponible en: http://www.shcp.gob.mx/POLITICAFINANCIERA/FINANZASPUBLICAS/Estadisticas_Oportunas_Finanzas_Publicas/Paginas/ unica2.aspx

“Estrategia Digital Nacional”, Diario Oficial de la Federación, 1 de noviembre, 2013.

Fernández, Mariana Cecilia, "Foucault y la gubernamentalidad en las sociedades de seguridad”, Question, vol. 1, núm. 44. Disponible en: http://sedici.unlp.edu.ar/ bitstream/handle/10915/43754/Documento_completo.pdf?sequence=1

García, Sergio y Ávila, Débora, Enclaves de riesgo: Gobierno neoliberal, desigualdad y control social, Madrid, Útiles.

Gramckow, Heike, Greene, Jack, Marshall, Ineke y Barão, Lisa, "Part 1: Crime, Poverty and the Police", Addressing the Enforcement Gap to Counter Crime: Investing in Public Safety, the Rule of Law and Local Development in Poor Neighborhoods, International Bank for Reconstruction and Development of The World Bank, 2016. Disponible en: http://documents.worldbank.org/curated/ en/142491467989521605/pdf/105089-WP-PUBLIC-Crime-and-Enforcement-Part1.pdf

Institute for Economics and Peace, "Mexico. Índice de Paz 2016”, Institute for Economics and Peace. Disponible en: http://economicsandpeace.org/wp-content/ uploads/2016/04/\%C3\%8Dndice-de-Paz-M\%C3\%A9xico-2016_ES.pdf

Instituto Nacional de Estadística y Geografía, "Encuesta Nacional sobre la Dinámica de las Relaciones en los Hogares, 2016”, Instituto Nacional de Estadística y Geografía. Disponible en: http://www.beta.inegi.org.mx/proyectos/enchogares/ especiales/endireh/2016/

Instituto Nacional de Estadística y Geografía, "Encuesta Nacional sobre Disponibilidad y Uso de las Tecnologías de la Información en los Hogares 2016”, Instituto Nacional de Estadistica y Geografía. Disponible en: http://www.beta.inegi.org.mx/ proyectos/enchogares/regulares/dutih/2016/ 
Instituto Nacional de Estadística y Geografía, “Encuesta Nacional de Victimización y Percepción de la Seguridad Pública, 2017”, Boletín de Prensa número 417/17, 26 de septiembre, 2017. Disponible en: http://www.inegi.org.mx/saladeprensa/boletines/2017/envipe/envipe2017_09.pdf

Instituto Nacional de Estadística y Geografía, Mortalidad, Proyectos estadísticos, Instituto Nacional de Estadistica y Geografía. Disponible en: http://www.beta.inegi. org.mx/proyectos/registros/vitales/mortalidad/

Instituto Nacional de Psiquiatría Ramón de la Fuente Muñiz, Encuesta Nacional de Consumo de Drogas, Alcohol y Tabaco (2016-2017): Reporte drogas, 2017. Disponible en: https://drive.google.com/file/d/1zIPBiYB3625GBGIW5BX0TT_YQN73eWhR/ view

Jaitman, Laura y Guerrero CompeÁn, Roberto, "Closing Knowledge Gaps: Toward Evidence-Based Crime Prevention Policies in Latin America and the Caribbean”, Inter-American Development Bank, Institutional Capacity of State Division, octubre, 2015. Disponible en: http://idbdocs.iadb.org/wsdocs/getdocument. aspx?docnum=39898005

LARENAS Álvarez, AngIE, "La confluencia entre estudios críticos de seguridad y seguridad humana: las dinámicas de inclusión y superación”, Relaciones Internacionales, núm. 23. Disponible en: https://revistas.uam.es/rrii/article/view/5180

Le Clerco Ortega, Juan Antonio y Sánchez Lara, Gerardo Rodríguez, "Índice Global de Impunidad 2016”, Universidad de Las Américas de Puebla. Disponible en: http:// www.udlap.mx/igimex/assets/files/igimex2016_ESP.pdf

Le Clerco Ortega, Juan Antonio y Sánchez Lara, Gerardo Rodríguez, "Índice Global de Impunidad 2017”, Universidad de Las Américas de Puebla. Disponible en: http:// www.udlap.mx/cesij/files/IGI-2017_esp.pdf

"Ley General del Sistema Nacional Anticorrupción”, Diario Oficial de la Federación, 18 de julio, 2016.

“Ley de Seguridad Interior”, Diario Oficial de la Federación, 21 de diciembre, 2017.

"Lineamientos para el otorgamiento de apoyos a las entidades federativas en el marco del Programa Nacional de Prevención del Delito", Diario Oficial de la Federación, 24 de enero, 2018.

“Los mexicanos vistos por sí mismos”, Nexos, 1 de noviembre, 2015. Disponible: https:// www.nexos.com.mx/?p=26737

Secretaría de Gobernación, Manual y Protocolo para la Elaboración de Políticas Públicas de Derechos Humanos Conforme a los Nuevos Principios Constitucionales, México, Secretaría de Gobernación, 2014.

Melchor Aguilar, Jaime, Martínez Revilla, Anel, "Los Sistemas de Investigación en México”, Cinta de Moebio, núm. 14. Disponible en: http://www.moebio.uchile.cl/14/ melchor.htm

Oficina de las Naciones Unidas contra la Droga y el Delito, "Manual sobre la aplicación eficaz de las Directrices para la prevención del delito”, Nueva York, Oficina de las Naciones unidas contra la Droga y el Delito, 2011. Disponible en: https:// 
www.unodc.org/documents/justice-and-prison-reform/crimeprevention/Handbook_on_the_Crime_Prevention_Guidelines_Spanish.pdf

Organización PARA la CoOPERAción y el Desarrollo Económicos, "La situación de México", Panorama de la Sociedad 2016. Disponible en: https://www.oecd.org/mexico/ sag2016-mexico.pdf

PARdinas, JuAn, ImPunidad, "Corrupción y Competitividad. Indice de Competitividad Nacional 2015”, La corrupción en Mexico: Transamos y no avanzamos, México, Instituto Mexicano para la Competitividad, 2015. Disponible en: http://imco. org.mx/indices/documentos/2015_ICI_Libro_La\%20corrupcion_en_Mexico. pdf

Pasin, Julia, “Complejo tutelar y prevención social del delito. Rupturas y continuidades”, $V$ Jornadas de Jóvenes Investigadores del Instituto de Investigaciones, Argentina, Instituto de Investigaciones Gino Germani de la Universidad de Buenos Aires, 2009.

PÉreZ de Armiño, Karlos, “¿Más allá de la seguridad humana? Desafíos y aportes de los estudios críticos de seguridad”, Cursos de derecho internacional y relaciones internacionales de Vitoria-Gasteiz, núm. 1, 2011.

“Plan Nacional de Desarrollo 2013-2018”, Diario Oficial de la Federación, 20 de mayo, 2013.

"Presupuesto de Egresos de la Federación para el Ejercicio Fiscal 2017", Diario Oficial de la Federación, 30 de noviembre, 2016.

"Programa Especial de Ciencia, Tecnología e Innovación 2014-2018”, Diario Oficial de la Federación, 30 de julio, 2014.

“Programa Gobierno Cercano y Moderno 2013-2018”, Diario Oficial de la Federación, 30 de agosto, 2013.

“Programa Nacional de Derechos Humanos 2014-2018”, Diario Oficial de la Federación, 30 de abril, 2014.

"Programa Nacional de Prevención Social de la Violencia y la Delincuencia 2014-2018”, Diario Oficial de la Federación, 30 de abril, 2014.

“Programa Nacional de Seguridad Pública 2014-2018”, Diario Oficial de la Federación, 30 de abril, 2014.

RAngel Cortés, Víctor Manuel, "Iniciativa Mérida y la reproducción del derecho penal mexicano”, Reforma Judicial, núm. 25-26. Disponible en: https://revistas.juridicas.unam.mx/index.php/reforma-judicial/article/view/10451/12595

"Reglamento de la Ley General para la Prevención Social de la Violencia y la Delincuencia”, Diario Oficial de la Federación: 19 de septiembre, 2014.

Rodríguez, CÉSAR y Uprimny, Rodrigo, “¿Justicia para todos o seguridad para el mercado?”, en La falacia neoliberal. Critica y alternativas, Colombia, Universidad Nacional de Colombia, 2003.

Rojas, Diana Marcela, "El Taller del Imperio Global: Análisis De La Intervención De Estados Unidos En Colombia (1998-2008)”, Análisis Político, vol. 22, núm. 65, 
2009. Disponible en: https://revistas.unal.edu.co/index.php/anpol/article/ view/45962/47517

SÁnchez, Carlos, Ríos, Humberto, "La economía del conocimiento como base del crecimiento económico en México”, Enl@ce: Revista Venezolana de Información, vol. 8, núm. 2.

Secretaría de Hacienda y CRÉdito Público, "Informe del Resultado de la Fiscalización Superior de la Cuenta Pública 2014 del Dictamen de la Auditoría de Desempeño 14-0-04100-07-0113 'Prevención Social de la Violencia y la Delincuencia”, Secretaría de Gobernación, 23 de octubre, 2015. Disponible en: https://www.asf. gob.mx/Trans/Informes/IR2014i/Documentos/Auditorias/2014_0113_a.pdf

Sistema Nacional de Seguridad Pública, "Datos Abiertos de Incidencia Delictiva, Acumulado Enero-Octubre 2017, Secretariado Ejecutivo. Disponible en: http://secretariadoejecutivo.gob.mx/incidencia-delictiva/incidencia-delictiva-datos-abiertos.php

Sistema Nacional de Seguridad Pública, "Incidencia delictiva del fuero común, Informe de víctimas de homicidio, secuestro y extorsión”, Secretariado Ejecutivo. Disponible en: http://www.secretariadoejecutivo.gob.mx/incidencia-delictiva/incidencia-delictiva-victimas.php\#

Silva Senarqué, Santos Alfonso, Control social, neoliberalismo y derecho penal, parte 3, Perú, UNMSM, 2002.

Silvio Donolo, Danilo, “Triangulación: Procedimiento incorporado a nuevas metodologías de investigación”, Revista digital Universitaria unAM, vol. 10, núm. 8. Disponible en: http://www.revista.unam.mx/vol.10/num8/art53/art53.pdf

Sistema Nacional de Seguridad Pública, "Datos Abiertos de Incidencia Delictiva, Acumulado enero-octubre, 2017. Disponible en: http://secretariadoejecutivo.gob.mx/ incidencia-delictiva/incidencia-delictiva-datos-abiertos.php

Transparency International, Corruption Perceptions Index 2016, Transparency International. Disponible en: https://www.transparency.org/news/feature/ corruption_perceptions_index_2016\#table

Torrego-Egido, Luis, “¿Investigación difusa o emancipatoria? Participación e inclusión en investigación educativa”, Magis Revista Internacional de Investigación en Educación, vol. 7, núm. 14.

United States Agency for International Development, "Prevención de la violencia y la delincuencia, 2016”. [Disponible en: https://www.usaid.gov/es/mexico/prevencion]

Uppsala Universitet, Uppsala Conflict Data Program, México, Department of Peace and Conflict Research, 2017. Disponible en: http://ucdp.uu.se/\#country/70

Valenzuela Reynaga, Rodolfo, Moreno Milanes, María Dolores y Peimbert Romero, MarLENE, "Indicadores de economía basada en el conocimiento en organizaciones de Cd. Obregón, Sonora, México”, Revista El Buzón de Pacioli, número especial 74. Disponible en: http://www.itson.mx/publicaciones/pacioli/Documents/ no74/17.-_indicadores_de_economia_basada_en_el_conocimiento___.pdf 
VARgas Beal, XavieR, ¿Cómo hacer investigación cualitativa? Una guía práctica para saber qué es la investigación en general y cómo hacerla, con énfasis en las etapas de la investigación cualitativa, México, Etxeta, 2011.

World ECONOMic Forum, "Networked Readiness Index 2016". World Economic Forum. Disponible en: http://reports.weforum.org/ global-information-technology-report-2016/economies/\#economy=MEX

World Health Organization, World health statistics 2017: monitoring health for the sDgs, Sustainable Development Goals, Geneva, World Health Organization, 2017. Disponible en: http://apps.who.int/iris/bitstream/10665/255336/1/97892 41565486-eng.pdf?ua=1 